\title{
Changes in smoking status, mental state and plasma clozapine concentration: retrospective cohort evaluation
}

\author{
Inti Qurashi, ${ }^{1}$ Paul Stephenson, ${ }^{1}$ (1) Chitra Nagaraj, ${ }^{2}$ Simon Chu, ${ }^{3}$ Richard Drake, ${ }^{4}$ Lewis Couchman, ${ }^{5}$ \\ Robert Flanagan ${ }^{6}$
}

BJPsych Bulletin (2019) 43, 271-274, doi:10.1192/bjb.2019.50

\begin{abstract}
${ }^{1}$ High Secure Services, Ashworth High Secure Hospital, UK; ${ }^{2}$ Low Secure Servies Hollins Park Hospital, UK; ${ }^{3}$ Department of Psychology, University of Central Lancashire, UK; ${ }^{4}$ Division of Psychology and Mental Health, University of Manchester, UK; ${ }^{5}$ Therapeutic Drug Monitoring, Analytical Services International, UK; ${ }^{6}$ Toxicology, King's College Hospital NHS Foundation Trust, UK

Correspondence to Dr Paul Stephenson (paul.stephenson2@merseycare.nhs.uk)

First received 24 Jan 2019, final revision 26 Apr 2019, accepted 15 May 2019

(c) The Authors 2019. This is an Open Access article, distributed under the terms of the Creative Commons Attribution-NonCommercialNoDerivatives licence (http:// creativecommons.org/licenses/ by-nc-nd/4.0/), which permits noncommercial re-use, distribution, and reproduction in any medium, provided the original work is unaltered and is properly cited. The written permission of Cambridge University Press must be obtained for commercial re-use or in order to create a derivative work.
\end{abstract}

Aims and method To investigate the percentage of patients who commenced smoking after transferring out of a non-smoking forensic psychiatric unit, the corresponding clozapine dose adjustments, the effects on plasma clozapine/ norclozapine concentrations and observed changes in mental state. We reviewed the notes and plasma clozapine/norclozapine concentrations of 46 patients transferred to medium secure units between July 2008 and December 2013.

Results Thirty-five patients commenced smoking. Their median clozapine dose was increased by $50 \mathrm{mg} / \mathrm{d}$. In the non-smokers, the median clozapine dose remained unchanged. Plasma clozapine/norclozapine concentrations were significantly reduced in smokers despite dosage adjustment. Eighteen patients experienced deterioration in mental state after transfer; almost all these patients were smokers.

Clinical implications Approximately three-quarters of patients who were non-smokers by virtue of being in a secure non-smoking environment commenced smoking after transfer. Monitoring of clozapine serum levels and assessment of mental state in the immediate period after a change in smoking status is indicated.

Declaration of interest None.

Keywords Clozapine; smoking; relapse.

\section{Clozapine use in schizophrenia}

Clozapine is the only antipsychotic licensed for use in patients with treatment-resistant schizophrenia. It has a narrow therapeutic index and pre-dose plasma clozapine concentrations between 0.35 and $0.60 \mathrm{mg} / \mathrm{L}$ are associated with optimal response, although patients may show such a response at lower or indeed higher plasma concentrations. ${ }^{1}$ Adverse effects are more likely at plasma concentrations above $0.6 \mathrm{mg} / \mathrm{L}^{2}$

\section{Clozapine metabolism}

Clozapine is primarily metabolised to its principal plasma metabolite $N$-desmethylclozapine (norclozapine) by the cytochrome P450 (CYP) isoenzyme CYP1A2, with possible additional contributions by CYP2C9, CYP2C19, CYP2D6 and CYP3A4. ${ }^{3,4}$ There are significant differences in plasma concentrations of clozapine between patients receiving the same dose, owing to factors including age, gender and exposure to tobacco smoke. ${ }^{5,6}$ The magnitude of the effects of the polycyclic aromatic hydrocarbons in tobacco smoke in increasing CYP1A2 activity are second only to dose on plasma clozapine concentrations. ${ }^{7}$ A systematic review of the effects of smoking cessation on plasma clozapine concentrations found clozapine dose reductions of between 30 and $40 \%$ were required to achieve pre-cessation concentrations. ${ }^{8}$ Neither the nicotine in cigarettes nor nicotine replacement therapy products have an effect on clozapine metabolism. $^{8}$

\section{Schizophrenia and smoking}

Patients with schizophrenia are more likely to smoke cigarettes, and in greater quantity, than the general population. ${ }^{9}$ An evaluation from a UK forensic psychiatric medium secure unit found $84 \%$ of in-patients were smokers before the introduction of a smoking ban, and cessation rates after smoking reduction programmes are less than $7 \% .^{10,11}$ 
Ashworth Hospital is a high-security psychiatric hospital serving the population of Northern England and Wales. The hospital has approximately 200 male in-patients who are detained under various sections of the Mental Health Act 1983; none have unsupervised community leave. The hospital became a non-smoking unit on 1 July 2008. Tobacco products are not permitted in the hospital. This is a policy that is effectively implemented as all hospital staff, patients and visitors are subject to rigorous security measures, including searches in and out of the hospital and x-ray scanning of personal possessions. Patients no longer requiring treatment in a high secure unit are transferred either to prison or to medium secure units. At the time of this evaluation, all the medium secure units to which patients were transferred permitted smoking.

\section{Clozapine and smoking}

We could find no published study that documented changes in smoking status and clozapine dose requirement after transferring out of a non-smoking psychiatric unit. We decided to investigate the percentage of patients who commenced smoking after transfer, the corresponding clozapine dose adjustments performed, the associated effects on plasma clozapine and norclozapine concentrations and observed changes in mental state.

\section{Method}

The service evaluation was registered within Mersey Care National Health Service (NHS) Foundation Trust. We identified patients prescribed clozapine who were discharged to medium secure units (both NHS and independent) from Ashworth Hospital from July 2008 to December 2013. Patients transferred to a medium secure unit on trial leave remain under the nominal care of the Responsible Clinician at Ashworth hospital and are routinely reviewed by clinical staff from Ashworth Hospital; trial leave is for a minimum period of 6 months and can be longer, allowing an opportunity to review case records and changes in smoking status. From the clinical notes we collected data on clozapine dose, plasma clozapine and norclozapine concentrations, smoking status and mental state in the 6 months pre-transfer and up to 12 months post-transfer. Patients who were either discharged without a period of trial leave, or remitted directly to court or prison were excluded. Plasma clozapine and norclozapine concentrations were monitored as necessary to help guide clozapine dose adjustment. The last clozapine plasma measurement on file was used as a measure of post-transfer plasma clozapine and norclozapine. Information routinely recorded when requesting plasma clozapine assays include the time and date of sample, time and date of last clozapine dose, clozapine dose (milligrams per day), mode of administration (tablets or suspension), duration of clozapine treatment, age (years), gender, body weight (kilograms), smoking status (yes/no) and other relevant information that could aid interpretation of the result, such as co-prescribed drugs and the reason for the assay request.

Statistical significance of analysed data was calculated using paired $t$-tests and Fisher's exact test as appropriate.

\section{Ethical approval}

The service evaluation was registered within Mersey Care NHS Foundation Trust. Ethical approval was not required; no individual patient is described.

\section{Results}

Forty-six male patients with complete data were identified. All had a diagnosis of schizophrenia (ICD-10 F20). ${ }^{12}$ Ethnicity was White British $(n=27)$, White Irish $(n=2)$, Black Caribbean $(n=3)$, Black African $(n=2)$, other Black background $(n=4)$, Pakistani $(n=2)$, Indian $(n=1)$, other Asian background $(n=1)$, and other mixed background $(n=4)$. The median age at the start of trial leave was 37.1 years (range, $24.8-56.0$ years).

Plasma clozapine and norclozapine concentrations had been measured in all patients before transfer (median time before transfer, 89.5 days; range, 1-315 days). The median number of plasma clozapine assays performed after transfer to a medium secure unit was 2 (range, 1-9). Plasma clozapine was first measured on average 45 days post-transfer (range, 2-345 days). A total of $72 \%$ of patients had plasma clozapine and norclozapine measured within 1 month of starting trial leave; this increased to $87 \%$ of patients at 3 months post-transfer.

Thirty-five patients (76\%) commenced smoking after transfer and eleven (24\%) remained non-smokers (Table 1). The average duration of follow-up was 6.6 months in both the smoking and non-smoking groups.

In the smoking group the median clozapine dose before transfer was $275 \mathrm{mg} / \mathrm{d}$ and this increased to $350 \mathrm{mg} / \mathrm{d}$ after

Table 1 Comparison of pre- and post-transfer data: clozapine dose $(\mathrm{mg} / \mathrm{d})$ and clozapine concentration (mg/L)

\begin{tabular}{|c|c|c|c|c|c|c|}
\hline & \multicolumn{3}{|c|}{ Smokers $(N=35)$} & \multicolumn{3}{|c|}{ Non-smokers $(N=11)$} \\
\hline & Pre-transfer & Post-transfer & $P$-value ${ }^{a}$ & Pre-transfer & Post-transfer & $P$-value ${ }^{a}$ \\
\hline Median (range) clozapine dose (mg/d) & $275(150-550)$ & $350(175-750)$ & & $250(125-500)$ & $250(200-400)$ & \\
\hline Mean (s.d.) clozapine dose (mg/d) & $306(101)$ & $376(133)$ & $<0.01$ & $270(104)$ & $282(67)$ & 0.54 \\
\hline $\begin{array}{l}\text { Mean (s.d.) plasma clozapine } \\
\text { concentration }(\mathrm{mg} / \mathrm{L})\end{array}$ & $0.58(0.20)$ & $0.43(0.15)$ & $<0.01$ & $0.47(0.16)$ & $0.40(0.14)$ & 0.11 \\
\hline $\begin{array}{l}\text { Mean (s.d.) plasma norclozapine } \\
\text { concentration (mg/L) }\end{array}$ & $0.34(0.12)$ & $0.28(0.12)$ & $<0.01$ & $0.33(0.10)$ & $0.29(0.11)$ & 0.03 \\
\hline
\end{tabular}

a. Two-tailed paired $t$-test. 
transfer; an increase of $27 \%$. The median dose increase was $50 \mathrm{mg} / \mathrm{d}$ (range, $75 \mathrm{mg} / \mathrm{d}$ decrease to $375 \mathrm{mg} / \mathrm{d}$ increase) and there was a statistically significant decrease in both plasma clozapine concentration and plasma norclozapine concentration after transfer in the smoking group. In the non-smoking group the median prescribed dose before transfer was 250 $\mathrm{mg} / \mathrm{d}$ and this remained unchanged after transfer (range, $100 \mathrm{mg} / \mathrm{d}$ decrease to $125 \mathrm{mg} / \mathrm{d}$ increase). We found no plasma clozapine concentrations $>1 \mathrm{mg} / \mathrm{L}$ in either the smoking or non-smoking group post-transfer.

Mental state deterioration was defined as either a worsening of residual symptoms after transfer or emergence of new psychopathology. Eighteen (39\%) patients showed evidence of deterioration in their mental state and of these, all but one were in the smoking group $(P<0.05$; Fisher's exact test). The median time to mental state changes was 54.5 days after transfer as recorded in the clinical notes (range, 6-175 days). Ten patients, all within the smoking group, experienced an exacerbation of psychotic symptoms (Table 2). For three patients who experienced increased psychotic symptoms, the trial leave period was extended as a consequence. Notably, no patients in the non-smoking group experienced either a worsening of psychotic symptoms or emergence of new psychopathology. The remaining eight patients, seven of which were in the smoking group and one in the non-smoking group, experienced non-psychotic changes in mental state, including increased self-harm, sexual disinhibition, amotivation, agitation and anxiety. In all cases $(N=18)$, the mental states of these patients improved with an increase in clozapine dose and no patients required readmission to the high-security psychiatric hospital as a consequence of a change in their mental status.

\section{Discussion}

A not unexpected finding was that most patients commenced smoking despite being provided with nicotine replacement therapy and smoking cessation advice both before and after transfer. Studies have shown that enforced tobacco abstinence in secure environments has little effect on postrelease smoking status. ${ }^{13,14}$

There is an established association between tobacco smoking and the pharmacokinetics of clozapine, with higher

\begin{tabular}{|lr|}
\hline Table 2 Symptoms experienced after transfer & \\
Psychotic symptoms (10 patients) & 7 \\
\hline Paranoid delusions & 3 \\
\hline Auditory hallucinations & 1 \\
\hline Religious delusions & 1 \\
\hline Delusional memory & 3 \\
\hline Non-psychotic symptoms (8 patients) & 3 \\
\hline Agitation & 1 \\
\hline Anxiety & 1 \\
\hline Amotivation & 1 \\
\hline Worsening self-harm & \\
\hline Sexual disinhibition & \\
\hline
\end{tabular}

clozapine doses generally required in smokers when compared with non-smokers. A previous evaluation of plasma clozapine concentrations before and after implementation of a hospital-wide smoking ban found a mean increase in plasma clozapine of $36 \%$ in non-smokers when outliers were excluded. ${ }^{15}$ We found a mean clozapine dose increase of $23 \%$ (median increase, $27 \%$ ) occurred in smokers prescribed clozapine and this is less than the $40 \%$ dose increase recommended where one or more packs of cigarettes are used per day ${ }^{16}$ and less than the recommendation to increase the clozapine dose by a factor of 1.5 over a period of $2-4$ weeks. ${ }^{17}$ This difference may be because of the effect of restricted time periods off the ward for patients in our sample, who were in a medium secure unit, which is likely to have limited their consumption of tobacco. However, it may also be that clinical teams were cautious in increasing clozapine doses, or patients were reluctant to take more clozapine, given the observed changes in mental state in the smoking group.

We found that receiving clinical teams were aware of the association between plasma clozapine and smoking status and the need to proactively monitor and adjust doses accordingly. As noted earlier, $72 \%$ of patients had plasma clozapine and norclozapine measured within 1 month of starting trial leave and this increased to $87 \%$ of patients at 3 months posttransfer. There were nevertheless adverse changes in the mental state of almost half of patients who had recommenced smoking. It is possible these symptoms were secondary to the observed reduction in plasma clozapine concentrations. Previous studies investigating the effects of smoking on mental state have focused on patients with neurosis rather than psychosis. ${ }^{18} \mathrm{We}$ found that most patients experiencing changes in mental state had psychotic symptoms, although this may reflect the symptoms of this particular population from a high secure psychiatric unit. However, structured rating scales were not used in our survey given the nature of this evaluation.

Our study population was of a modest size and data were collected over a minimum follow-up period of 6 months, allowing ample time for changes to clozapine dosing schedules and changes in mental status to have been observed. A limitation of this study is our use of case notes as a source of patient data as case notes do not always include all data that would otherwise have been collected systematically via a structured interview. Our recording of patient mental status before and after smoking resumption is therefore open to assessment bias. Additionally, the post-transfer plasma clozapine and norclozapine concentrations used in this study were not necessarily performed before the recorded dose adjustments. Given the nature of this study (i.e. a service evaluation), we did not control for clozapine dose when measuring the effect of smoking on clozapine and norclozapine plasma concentrations. It should be noted that the non-smokers had a statistically significant lower norclozapine plasma concentration after transfer; we are unable to explain this finding. Finally, we cannot be certain that the non-smoking patients were entirely abstinent as we relied on patient notes to classify smoking status; we did not measure plasma or urine cotinine to confirm smoking status.

This evaluation is a reminder to clinicians that changes in smoking status may cause marked changes in clozapine 
plasma concentration, and require substantial adjustments in clozapine dosing. We anticipate this evaluation will assist clinicians in the management of patients prescribed clozapine who commence smoking after a period of abstinence and advocate close monitoring of plasma clozapine concentrations, clozapine dose and mental status in patients who change their smoking status, to minimise the risk of relapse.

\section{About the authors}

Inti Qurashi is a consultant forensic psychiatrist in High Secure Services at Ashworth High Secure Hospital, Mersey Care NHS Foundation Trust, UK. Paul Stephenson is a consultant forensic psychiatrist in High Secure Services at Ashworth High Secure Hospital, Mersey Care NHS Foundation Trust, UK. Chitra Nagaraj is a consultant forensic psychiatrist in Low Secure Services at Hollins Park Hospital, North West Boroughs NHS Foundation Trust, UK. Simon Chu is a senior lecturer in the Department of Psychology at the School of Psychology, University of Central Lancashire, UK. Richard Drake is a senior lecturer and honorary consultant at the Division of Psychology and Mental Health, University of Manchester, UK. Lewis Couchman is Facility Director of Therapeutic Drug Monitoring at Analytical Services International, UK. Robert Flanagan is a consultant clinical scientist and Director of the Toxicology Unit at King's College Hospital NHS Foundation Trust, UK.

\section{References}

1 Taylor D, Paton C, Kerwin R. The South London and Maudsley NHS Foundation Trust and Oxleas Foundation Trust Prescribing Guidelines (12th edn). Informa Healthcare, 2015.

2 Rajkumar AP, Poonkuzhali B, Kuruvilla A, Jacob M, Jacob KS. Clinical predictors of serum clozapine levels in patients with treatment-resistant schizophrenia. Int Clin Psychopharmacol 2013; 28: 50-6.

3 Pirmohamed M, Williams D, Madden S, Templeton E, Park BK. Metabolism and bioactivation of clozapine by human liver in vitro. J Pharmacol Exp Ther 1995; 272: 984-90.

4 Chetty M, Murray M. CYP-mediated clozapine interactions: how predictable are they? Curr Drug Metab 2007; 8: 307-13.

5 Bowskill S, Couchman L, MacCabe JH, Flanagan RJ. Plasma clozapine and norclozapine in relation to prescribed dose and other factors in patients aged 65 years and over: data from a therapeutic drug monitoring service, 1996-2010. Hum Psychopharmacol 2012; 27: 277-83.

6 Bersani FS, Capra E, Minichino A, Pannese R, Girardi N, Marini I, et al. Factors affecting inter individual differences in clozapine response: a review and case report. Hum Psychopharmacol 2011; 26: 177-87.

7 Olsson E, Edman G, Bertilsson L, Hukic DS, Lavebratt C, Eriksson SV et al. Genetic and clinical factors affecting plasma clozapine concentration. Prim Care Companion CNS Disord 2015; 17. doi:10.4088/ PCC.14m01704.

8 Lowe EJ, Ackman ML. Impact of tobacco smoking cessation on stable clozapine or olanzapine treatment. Ann Pharmacother 2010; 44: 727-32.

9 Lohr JB, Flynn K. Smoking and schizophrenia. Schizophr Res 1992; 8: 93-102.

10 Meiklejohn C, Sanders K, Butler S. Physical health in medium secure services. Nurs Stand 2003; 17: 33-7.

$11 \mathrm{Wu}$ BJ, Lan TH. Predictors of smoking reduction outcomes in a sample of 287 patients with schizophrenia spectrum disorders. Eur Arch Psychiatry Clin Neurosci 2017; 267: 63-72.

12 World Health Organisation. Classifications of Mental and Behavioural Disorder: Clinical Descriptions and Diagnostic Guidelines (ICD-10). World Health Organisation, 1992

13 Clarke JG, Stein LA, Martin RA, Martin SA, Parker D, Lopes CE, et al. Forced smoking abstinence: not enough for smoking cessation. JAMA Intern Med 2013; 173: 789-94.

14 Lincoln T, Tuthill RW, Roberts CA, Kennedy S, Hammett TM,

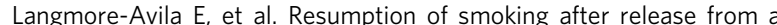
tobacco-free correctional facility. J Correct Health Care 2009; 15: 190-6.

15 Murayama-Sung L, Ahmed I, Goebert D, Alaimalo E, Sung H. The impact of hospital smoking ban on clozapine and norclozapine levels. J Clin Psychopharmacol 2011; 31: 124-6.

16 Rostami-Hodjegan A, Amin AM, Spencer EP, Lennard MS, Tucker GT, Flanagan RJ. Influence of dose, cigarette smoking, age, sex, and metabolic activity on plasma clozapine concentrations: a predictive model and nomograms to aid clozapine dose adjustment and to assess compliance in individual patients. J Clin Psychopharmacol 2004; 24: 70-8.

17 de Leon J. Atypical antipsychotic dosing: the effect of smoking and caffeine. Psychiatr Serv 2004; 55: 491-3.

18 Taylor G, McNeill A, Girling A, Farley A, Lindson-Hawley N, Aveyard P. Change in mental health after smoking cessation: systematic review and meta-analysis. BMJ 2014; 348: g1151.

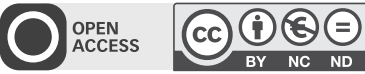

